\title{
LIMIT DISTRIBUTIONS OF THE MINIMAX OF INDEPENDENT IDENTICALLY DISTRIBUTED RANDOM VARIABLES $\left({ }^{1}\right)$
}

\author{
BY \\ HERMAN CHERNOFF AND HENRY TEICHER
}

Summary. The class of limiting distributions of the normalized minimax (or maximin) of independent identically distributed random variables is obtained and the domains of attraction of the three limiting types are characterized. Asymptotic independence of the minimax and maximin is also demonstrated.

1. Introduction. Consider a sequence of independent identically distributed random variables on some probability space with $P$ the probability measure thereupon. These will be double indexed in the fashion

$$
X_{11} ; X_{12}, X_{22}, X_{21} ; X_{13}, X_{23}, X_{33}, X_{32}, X_{31} ; \cdots
$$

and engender a sequence of random matrices of order $n$

$$
\chi_{n}=\left(\begin{array}{cccc}
X_{11} & X_{12} & \cdots & X_{1 n} \\
\vdots & \vdots & & \vdots \\
X_{n 1} & X_{n 2} & \cdots & X_{n n}
\end{array}\right), \quad n=1,2, \cdots,
$$

which, in turn, give rise to two sequences of random variables

$$
\begin{aligned}
W_{n} & =\min _{i} \max _{j} X_{i j}, \\
Z_{n} & =\max _{j} \min _{i} X_{i j} .
\end{aligned}
$$

Denote by $F$ the common cumulative distribution function (c.d.f.) of the $X_{i j}$. We seek the classes $\mathscr{L}_{1}$ and $\mathscr{L}_{2}$ of limiting distributions of $W_{n}$ and $Z_{n}$ (suitably normalized) as well as conditions insuring that the normalized $W_{n}$ or $Z_{n}$ based on a stipulated $F$, actually converge in law to a particular limiting distribution. In view of the relationship $Z_{n}=-\min _{i} \max _{j}\left(-X_{j i}\right)$, it suffices to deal with $W_{n}$. Clearly, $\mathscr{L}_{i}, i=1,2$, is independent of the initial indexing procedure.

The problem is redolent of that of the class of limiting distributions of the normalized maximum of independent, indentically distributed variables.

Received by the editors April 22, 1964.

( 1 ) This work was prepared with the partial support of the Office of Naval Research Contract Nonr-225(72) at Stanford University. Reproduction in whole or in part is permitted for any purpose of the United States Government. 
Here, it will be recalled [3] that the class of limiting distributions comprises the three distribution types $(\alpha>0)$ :

$$
\begin{aligned}
L_{1 \alpha}(x) & =\exp \left\{-(-x)^{\alpha}\right\}, & & x \leqq 0, \\
& =1, & & x>0, \\
L_{2 \alpha}(x) & =0, & & x \leqq 0, \\
& =\exp \left\{-x^{-\alpha}\right\}, & & x>0, \\
L_{3}(x) & =\exp \left\{-e^{-x}\right\} . & &
\end{aligned}
$$

In the case of the minimum of independent, identically distributed random variables, the three (nondegenerate) limiting types are given by $L_{j}^{*}(x)$ $=1-L_{j}(-x), j=1,2,3$.

It follows readily from (2.0) that $W_{n}$, like the maximum, converges almost certainly to $+\infty$ or $c$ according as $F(x)<1$, all $x$ or $F(c-\epsilon)<1=F(c+\epsilon)$, all $\epsilon>0$. On the other hand, the only nondegenerate limiting types possible in the case of $W_{n}$ are $L_{j}^{*}, j=1,2,3$, namely, those corresponding to the minimum of independent identically distributed chance variables.

2. Limiting distributions. Let $\left\{a_{n}, b_{n}, n=1,2, \cdots\right\}$ constitute two sequences of constants with $a_{n}>0$. Evidently,

$$
P\left\{\frac{1}{a_{n}}\left(W_{n}-b_{n}\right)<x\right\}=1-\left[1-F^{n}\left(a_{n} x+b_{n}\right)\right]^{n} .
$$

As a first step in the direction adumbrated, we prove (letting $\mathscr{L}(W)$ denote generically the c.d.f. or "law" of a random variable $W$ )

Lemma 1. A necessary and sufficient condition that $\mathscr{L}\left[\left(W_{n}-b_{n}\right) / a_{n}\right]$ converge to a nondegenerate limiting distribution is that there exist an extended real-valued, nondecreasing, right-continuous function $\gamma(x)$ with $\gamma(\infty)=\infty$, $\gamma(-\infty)=-\infty$ such that for $x$ in the (nonempty) set of points at which $\gamma(x)$ is continuous and finite,

$$
F\left(a_{n} x+b_{n}\right)=1-\frac{\log n-\gamma(x)+o(1)}{n} .
$$

Proof. Suppose that (2.0) converges to a nondegenerate c.d.f. $\Gamma(x)$. Clearly, $F^{n}\left(a_{n} x+b_{n}\right)=o(1)$ and for $0<\Gamma(x)<1$

$$
n \log \left[1-F^{n}\left(a_{n} x+b_{n}\right)\right]=[1+o(1)] \log [1-\Gamma(x)],
$$

implying

$$
-n F^{n}\left(a_{n} x+b_{n}\right)=[1+o(1)] \log [1-\Gamma(x)] .
$$

Thus, if

$$
\gamma(x)=\log (-\log [1-\Gamma(x)])
$$


we have

$$
\log n+n \log F\left(a_{n} x+b_{n}\right)=\gamma(x)+o(1) .
$$

This implies $n\left[1-F\left(a_{n} x+b_{n}\right)\right]^{2}=o(1)$ and consequently also

$$
\log n-n\left[1-F\left(a_{n} x+b_{n}\right)\right]=\gamma(x)+o(1)
$$

which is tantamount to (2.1).

Retracing the argument in the reverse direction, (2.1) implies that (2.0) converges to $\Gamma(x)=1-\exp \left\{-e^{\gamma(x)}\right\}$ at all continuity points in the domain where $\gamma$ is finite, and thus at all continuity points.

This leads to

THEOREM $1\left(^{2}\right)$. The class $\mathscr{L}_{1}$ of nondegenerate limiting distributions of the normalized minimax $W_{n}$ of independent identically distributed random variables (as in (1.1)-(1.3)) contains only the types $(\alpha>0)$

$$
\begin{aligned}
L_{1 \alpha}^{*}(x) & =0, & & x<0, \\
& =1-\exp \left\{-x^{\alpha}\right\}, & & x \geqq 0, \\
L_{2 \alpha}^{*}(x) & =1-\exp \left\{-(-x)^{-\alpha}\right\}, & & x<0, \\
& =1, & & x \geqq 0, \\
L_{3}^{*}(x) & =1-\exp \left\{-e^{x}\right\} . & &
\end{aligned}
$$

Proof. Suppose that (2.0) converges to a nondegenerate limiting distribution $\Gamma$, whence according to the lemma, (2.1) holds.

Define, for any real $\delta$,

$$
m=m_{n}(\delta)=\{\text { greatest integer } \leqq n(1+\delta / \log n)\} .
$$

Then, if $A$ denotes the set of points at which $\gamma(x)$ is continuous and finite, for $x \in A$ we have from (2.1),

$$
\begin{aligned}
F\left(a_{m} x+b_{m}\right) & =1-\frac{\log m-\gamma(x)}{m}+o\left(m^{-1}\right) \\
& =1-\frac{\log n+\delta(\log n)^{-1}+o\left[(\log n)^{-1}\right]-\gamma(x)}{n\left[1+\delta(\log n)^{-1}\right]}+o\left(n^{-1}\right) \\
& =1-\frac{\log n-(\gamma(x)+\delta)+o(1)}{n}
\end{aligned}
$$

Let us call $(x, \gamma)$ a point of increase if $\gamma(x-\epsilon)<\gamma<\gamma(x+\epsilon)$ for all $\epsilon>0$. Then at every point of increase $(x, \gamma)$, the inverse function $h(\gamma)$ is

$\left(^{2}\right)$ Added in proof. Theorem 1 extends to rectangular matrices. 
uniquely defined and $h(\gamma)=x$. Further, let $G(x)$ denote an inverse function of $1-F(x)$ (any monotone version will do in spite of the ambiguity due to intervals where $F(x)$ is constant). It follows from (2.1) that for any sequence of numbers $\epsilon_{n} \rightarrow 0$ and point of increase $(h(\gamma), \gamma)$,

$$
\lim _{n \rightarrow \infty} \frac{1}{a_{n}}\left[G\left(\frac{\log n-\gamma+\epsilon_{n}}{n}\right)-b_{n}\right]=h(\gamma) .
$$

Let $m=m_{n}(\delta)$ be as in (2.4) and note that

$$
\frac{\log m-\gamma}{m}=\frac{\log n-(\gamma+\delta)+o(1)}{n} .
$$

Consequently, if $(h(\gamma+\delta), \gamma+\delta)$ and $(h(\gamma), \gamma)$ are points of increase, from (2.5) and (2.6)

$$
\begin{aligned}
& G\left(\frac{\log n-(\gamma+\delta)+o(1)}{n}\right) \\
& \quad=a_{n}[h(\gamma+\delta)+o(1)]+b_{n}=a_{m}[h(\gamma)+o(1)]+b_{m} .
\end{aligned}
$$

Applying the preceding to $\gamma_{0}, \gamma_{1}$, and $\gamma$, we have for all real $\delta$

$$
\begin{aligned}
& a_{n}[h(\gamma+\delta)+o(1)]+b_{n}=a_{m}[h(\gamma)+o(1)]+b_{m}, \\
& a_{n}\left[h\left(\gamma_{0}+\delta\right)+o(1)\right]+b_{n}=a_{m}\left[h\left(\gamma_{0}\right)+o(1)\right]+b_{m}, \\
& a_{n}\left[h\left(\gamma_{1}+\delta\right)+o(1)\right]+b_{n}=a_{m}\left[h\left(\gamma_{1}\right)+o(1)\right]+b_{m},
\end{aligned}
$$

whence,

$$
\frac{h(\gamma+\delta)-h\left(\gamma_{1}+\delta\right)}{h\left(\gamma_{1}+\delta\right)-h\left(\gamma_{0}+\delta\right)}=\frac{h(\gamma)-h\left(\gamma_{1}\right)}{h\left(\gamma_{1}\right)-h\left(\gamma_{0}\right)}
$$

provided all points involved correspond to points of increase of $\gamma$ and the denominators do not vanish.

The nondegeneracy of the limiting distribution implies that $h(\gamma)$ is not constant for $-\infty<\gamma<\infty$. Now suppose $h$ is constant on some proper subinterval $I$ of $-\infty<\gamma<\infty$, say $h(\gamma) \equiv \beta$ in $I$ while $h(\gamma) \neq \beta$ outside $I$. Then choosing $\gamma_{1} \in I, \gamma \notin I, \gamma_{0} \notin I$ and $\delta$ such that $\gamma_{0}+\delta \notin I$ but both $\gamma_{1}+\delta$ and $\gamma+\delta$ are in $I$ would controvert (2.8). Since at most countably many $\gamma$ values do not correspond to points of increase, such a choice can be made. Therefore $h$ is strictly monotone.

Next, suppose that $\gamma_{0}+i \delta, i=0,1, \cdots, k^{*}+1$ correspond to points of increase. Let $\gamma=\gamma_{1}+\delta, \gamma_{i}=\gamma_{0}+(i-1) \delta$ in (2.8) and verify inductively that for all integers $k, 1 \leqq k \leqq k^{*}$

$$
\frac{h\left(\gamma_{0}+(k+1) \delta\right)-h\left(\gamma_{0}+k \delta\right)}{h\left(\gamma_{0}+k \delta\right)-h\left(\gamma_{0}+(k-1) \delta\right)}=\frac{h\left(\gamma_{0}+2 \delta\right)-h\left(\gamma_{0}+\delta\right)}{h\left(\gamma_{0}+\delta\right)-h\left(\gamma_{0}\right)}=\lambda \quad \text { (say). }
$$

Thus, 


$$
h\left(\gamma_{0}+(k+1) \delta\right)-h\left(\gamma_{0}+k \delta\right)=\lambda^{k}\left[h\left(\gamma_{0}+\delta\right)-h\left(\gamma_{0}\right)\right], \quad 0 \leqq k \leqq k^{*},
$$

which necessitates

$$
\begin{aligned}
h\left(\gamma_{0}+k \delta\right) & =c_{1} \lambda^{k}+d_{1}, \quad 0 \leqq k \leqq k^{*} \text { for } \lambda \neq 1, \\
& =c_{1} \lambda+d_{1}, \quad 0 \leqq k \leqq k^{*} \text { for } \lambda=1 .
\end{aligned}
$$

Select an interval $\left(x_{1}, x_{2}\right)$ such that $-\infty<x_{1}<x_{2}<\infty$ and $-\infty<\gamma\left(x_{1}+\right)$ $<\gamma\left(x_{2}-\right)<\infty$. In the range $\gamma\left(x_{1}-\right)$ to $\gamma\left(x_{2}+\right)$ at most a countable set of $\gamma$ values do not correspond to points of increase. Hence, in arbitrary neighborhoods of $\gamma\left(x_{1}-\right)$ and $\gamma\left(x_{2}+\right)$ we can find $\gamma_{1}^{*}$ and $\gamma_{2}^{*}$ such that $\gamma_{1}^{*}<\gamma_{2}^{*}$ and all of the values $\gamma_{1}^{*}+j 2^{-k}\left(\gamma_{2}^{*}-\gamma_{1}^{*}\right), j=0,1,2, \cdots, 2^{k}, k=1$, $2, \cdots$, correspond to points of increase. From (2.9) and monotonicity, for $\gamma\left(x_{1}-\right)<\gamma<\gamma\left(x_{2}+\right)$,

$$
h(\gamma)=c e^{\gamma / \alpha}+d, \quad \alpha \neq 0
$$

or

$$
h(\gamma)=c \gamma+d .
$$

Letting $x_{1}$ decrease and $x_{2}$ increase so that $\gamma\left(x_{1}-\right) \rightarrow-\infty$ and $\gamma\left(x_{2}+\right) \rightarrow \infty$, it follows that (2.10) or (2.10)' holds for all $\gamma$. Note that $\gamma\left(x_{1}+\right)=-\infty$ for finite $x_{1}$ entails $\alpha>0$, and $c>0$ whereas $\gamma\left(x_{2}\right)=+\infty$ for finite $x_{2}$ requires $\alpha<0$, and $c<0$. Summarizing, we find three possibilities:

$$
\begin{aligned}
& h(\gamma)=c \gamma+d, \quad c>0, \\
& \gamma(x)=\frac{x-d}{c}, \quad-\infty<x<\infty, \quad c>0,
\end{aligned}
$$$$
h(\gamma)=c e^{\gamma / \alpha}+d, \quad \alpha>0, c>0,
$$

(ii)

$$
\begin{aligned}
& \gamma(x)=\alpha \log \frac{x-d}{c}, \quad x>d, \quad c>0, \quad \alpha>0, \\
& h(\gamma)=c e^{-\gamma / \alpha}+d, \quad \alpha>0, \quad c<0,
\end{aligned}
$$

$$
\gamma(x)=-\alpha \log \frac{x-d}{c}, \quad x<d, \quad c<0, \quad \alpha>0 .
$$

These correspond via (2.2) to the distribution types $L_{3}^{*}(x), L_{1 \alpha}^{*}(x), L_{2 \alpha}^{*}(x)$ respectively of (2.3) and the proof is therefore complete.

DEFinition. A c.d.f. $F$ will be said to belong to the minimax domain of attraction of a c.d.f. $\left({ }^{3}\right) L^{*}$ (in symbols, $F \in \mathscr{D}_{m M}\left(L^{*}\right)$ ) if there exist sequences $b_{n}$ and $a_{n}>0$ for which $\lim _{n \rightarrow \infty}\left(1-\left[1-F^{n}\left(a_{n} x+b_{n}\right)\right]^{n}\right)=L^{*}(x)$. In similar fashion, we may say that $F$ belongs to the "maximum" domain of

$\left({ }^{3}\right)$ Of course, if $F \in \mathscr{D}_{m M}\left(L^{*}(x)\right)$, then $F \in \mathscr{D}_{m M}\left(L^{*}(a x+b)\right)$ for every $a>0$ and real $b$ so it is the distribution "type" that is pertinent. 
attraction of $L$ (written $F \in \mathscr{D}_{M}(L)$ ), $F$ belongs to the "minimum" domain of attraction of $L^{*}$ (written $F \in \mathscr{D}_{m}\left(L^{*}\right)$ ) or $F$ belongs to the maximin domain of attraction of $L$ (symbolically, $F \in \mathscr{D}_{M m}(L)$ ) according as $\lim _{n \rightarrow \infty} F^{n}\left(\tilde{a}_{n} x+\tilde{b}_{n}\right)=L(x), \lim _{n \rightarrow \infty}\left(1-\left[1-F\left(\bar{a}_{n} x+\bar{b}_{n}\right)\right]^{n}\right)=L^{*}(x)$ or $\lim _{n \rightarrow \infty}\left(1-\left[1-F\left(a_{n}^{\prime} x+b_{n}^{\prime}\right)\right]^{n}\right)^{n}=L(x)$.

Next, for any c.d.f. $F$, define

$$
a_{n}^{*}=G\left(\frac{\log n-1}{n}\right)-G\left(\frac{\log n}{n}\right), \quad n \geqq 1,
$$

where $G$ is again an inverse function of $1-F$. Then $a_{m_{n}(b)}^{*}$ is implicitly defined by (2.12) and (2.4) and

$$
a_{m_{n}(\delta)}^{*}=G\left(\frac{\log n-1-\delta+\bar{\epsilon}_{n}^{\prime}}{n}\right)-G\left(\frac{\log n-\delta+\overline{\epsilon_{n}}}{n}\right)
$$

where $\overline{\epsilon_{n}}, \bar{\epsilon}_{n}^{\prime}$ are both $o(1)$ and depend on $\delta$.

As will be seen in the ensuing theorems, the domain to which $F$ is attracted depends on the limit of $a_{m_{n}(\delta)}^{*} / a_{n}^{*}$ or more precisely upon that of the related quantity $r_{n}(1, \delta)$ (see $(2.13)$ ). Define

$$
\begin{aligned}
r_{n}(\beta, \delta) & =r_{n}\left(\beta, \delta ; \epsilon_{n}, \epsilon_{n}^{\prime}\right) \\
& =\frac{1}{a_{n}^{*}}\left[G\left(\frac{\log n-\delta-\beta+\epsilon_{n}^{\prime}}{n}\right)-G\left(\frac{\log n-\delta+\epsilon_{n}}{n}\right)\right]
\end{aligned}
$$

where $\epsilon_{n}^{\prime}, \epsilon_{n}$ are sequences of real numbers approaching zero.

Theorem 2. In order that $F \in \mathscr{D}_{m M}\left(L_{3}^{*}\right)$ with $a_{n}=a_{n}^{*}$, it is necessary that

$$
\lim _{n \rightarrow \infty} r_{n}(\beta, \delta)=\beta \text { for all real } \beta, \delta \text { and } \epsilon_{n}=o(1)=\epsilon_{n}^{\prime} .
$$

Conversely, if $\lim r_{n}(\beta, \delta)$ exists $\left({ }^{4}\right)$ for all $\beta, \delta$ and $\epsilon_{n}^{\prime}=o(1)=\epsilon_{n}$ and if for all real $\delta$ and $\epsilon_{n}=o(1)=\epsilon_{n}^{\prime}$,

$$
\lim _{n \rightarrow \infty} r_{n}(1, \delta)=1 \text {, }
$$

then $F \in \mathscr{D}_{m M}\left(L_{3}^{*}\right)$ with $a_{n}=a_{n}^{*}$ and $b_{n}=G(\log n / n)$.

Necessity. (2.14) is a simple consequence of (2.6) and (2.10)'

Sufficiency. Set $a_{n}=a_{n}^{*}$. It follows directly from $(2.14)^{\prime}$ that

$$
\lim _{n \rightarrow \infty} \frac{1}{a_{n}}\left[G\left(\frac{\log n-\delta-k+\epsilon_{n}^{\prime}}{n}\right)-G\left(\frac{\log n-\delta+\epsilon_{n}}{n}\right)\right]=k
$$

( ${ }^{4}$ It is not supposed that this limit is independent of $\epsilon_{n}$ and $\epsilon_{n}^{\prime}$. Also, an example can be adduced to show that $(2.14)^{\prime}$ by itself is insufficient. 
for all $\epsilon_{n}=o(1)=\epsilon_{n}^{\prime}$, all real $\delta$ and positive integers $k$, hence for all negative integers $k$, whence

$$
\begin{aligned}
\lim _{n \rightarrow \infty} \frac{1}{a_{n}}\left[G\left(\frac{\log n-\delta+\epsilon_{n}^{\prime}}{n}\right)-G\left(\frac{\log n-\delta+\epsilon_{n}}{n}\right)\right] \\
=\lim _{n \rightarrow \infty} \frac{1}{a_{n}}\left[G\left(\frac{\log n-\delta+\epsilon_{n}^{\prime}}{n}\right)-G\left(\frac{\log n-\delta-k}{n}\right)\right. \\
\left.+G\left(\frac{\log n-\delta-k}{n}\right)-G\left(\frac{\log n-\delta+\epsilon_{n}}{n}\right)\right]=0
\end{aligned}
$$

for all real $\delta$ and all $\epsilon_{n}$, $\epsilon_{n}^{\prime}$ which are $o(1)$. This shows further that $\lim r_{n}\left(\beta, \delta ; \epsilon_{n}, \epsilon_{n}^{\prime}\right)$ is independent of $\epsilon_{n}$ and $\epsilon_{n}^{\prime}$.

Consider next the subsequence $r_{n^{\prime}}\left(\beta, \delta ; \epsilon_{n^{\prime}}, \epsilon_{n^{\prime}}^{\prime}\right)$ of $r_{n}\left(\beta, \delta ; \epsilon_{n}, \epsilon_{n}^{\prime}\right)$ where $n^{\prime}=m_{n}\left(\delta^{\prime}\right)$. This subsequence may be delineated as

$$
\frac{1}{a_{m_{n}\left(\delta^{\prime}\right)}}\left[G\left(\frac{\log n-\left(\delta+\delta^{\prime}\right)-\beta+\epsilon_{n}^{* \prime}}{n}\right)-G\left(\frac{\log n-\left(\delta+\delta^{\prime}\right)+\epsilon_{n}^{*}}{n}\right)\right]
$$

where $\epsilon_{n}^{* \prime}$ and $\epsilon_{n}^{*}$ are again $o(1)$. In view of (2.14)' and (2.15), this subsequence has the same limit as $r_{n}\left(\beta, \delta+\delta^{\prime} ; \epsilon_{n}, \epsilon_{n}^{\prime}\right)$. Thus, $\lim r_{n}(\beta, \delta)$ is independent of $\delta$ (as well as $\epsilon_{n}, \epsilon_{n}^{\prime}$ ). Consequently, for any positive integer $k$ and all real $\delta$

$$
\begin{aligned}
k \lim _{n \rightarrow \infty} & \frac{1}{a_{n}}\left[G\left(\frac{\log n-\delta-1 / k}{n}\right)-G\left(\frac{\log n-\delta}{n}\right)\right] \\
= & \sum_{i=0}^{k-1} \lim _{n \rightarrow \infty} \frac{1}{a_{n}}\left[G\left(\frac{\log n-\delta-(i+1) / k}{n}\right)-G\left(\frac{\log n-\delta-i / k}{n}\right)\right] \\
= & 1,
\end{aligned}
$$

which readily implies for all integers $j, k$, real $\delta$ and $\epsilon_{n}=o(1)=\epsilon_{n}^{\prime}$, that

$$
\lim _{n \rightarrow \infty} \frac{1}{a_{n}}\left[G\left(\frac{\log n-\delta-j / k+\epsilon_{n}^{\prime}}{n}\right)-G\left(\frac{\log n-\delta+\epsilon_{n}}{n}\right)\right]=j / k .
$$

Next, let $r_{i}^{\prime}\left(r_{i}^{\prime \prime}\right)$ be a monotone sequence of rationals converging from below (above) to the real number $\beta$. Then, from (2.16)

$$
r_{i}^{\prime} \leqq \lim _{n \rightarrow \infty} \frac{1}{a_{n}}\left[G\left(\frac{\log n-\beta-\delta+\epsilon_{n}^{\prime}}{n}\right)-G\left(\frac{\log n-\delta+\epsilon_{n}}{n}\right)\right] \leqq r_{i}^{\prime \prime}
$$

and consequently $\lim _{n \rightarrow \infty} r_{n}(\beta, \delta)=\beta$ for all real $\beta, \delta$ and sequences $\epsilon_{n}, \epsilon_{n}^{\prime}$ tending to zero. In particular, setting $b_{n}=G(\log n / n)$, for all real $x$

$$
\lim _{n \rightarrow \infty} \frac{1}{a_{n}}\left[G\left(\frac{\log n-x+\epsilon_{n}}{n}\right)-b_{n}\right]=x .
$$


Hence, for any $\epsilon>0$ and $n>N$,

$$
a_{n}(x-\epsilon)+b_{n} \leqq G\left(\frac{\log n-x+\epsilon_{n}}{n}\right) \leqq a_{n}(x+\epsilon)+b_{n}
$$

or equivalently,

$$
F\left[a_{n}(x-\epsilon)+b_{n}\right] \leqq 1-\frac{\log n-x+\epsilon_{n}}{n} \leqq F\left[a_{n}(x+\epsilon)+b_{n}\right]
$$

Thus, for $n>N^{*}$

$$
1-\frac{\log n-x+2 \epsilon+\epsilon_{n}}{n} \leqq F\left(a_{n} x+b_{n}\right) \leqq 1-\frac{\log n-x-2 \epsilon+\epsilon_{n}}{n}
$$

which is tantamount to $(2.1)$ for $\gamma(x)=x$ and therefore proves sufficiency.

Theorem 3. In order that $F \in \mathscr{D}_{m M}\left(L_{1 \alpha}^{*}\right)$ with $a_{n}=a_{n}^{*}\left(e^{1 / \alpha}-1\right)^{-1}$, it is necessary that

$$
\lim _{n \rightarrow \infty} r_{n}(\beta, \delta)=e^{\delta / \alpha}\left(e^{\beta / \alpha}-1\right)\left(e^{1 / \alpha}-1\right)^{-1}
$$

for all real $\beta, \delta$ and $\epsilon_{n}^{\prime}=o(1)=\epsilon_{n}$. Conversely, if $\lim r_{n}(\beta, \delta)$ exists $\left({ }^{4}\right)$ for all real $\beta, \delta$ and $\epsilon_{n}=o(1)=\epsilon_{n}^{\prime}$ and if for some $\alpha>0$ and all real $\delta$ and $\epsilon_{n}^{\prime}=o(1)=\epsilon_{n}$,

$$
\lim _{n \rightarrow \infty} r_{n}(1, \delta)=e^{\delta / \alpha} \text {, }
$$

then $F \in \mathscr{D}_{m M}\left(L_{1 \alpha}^{*}\right)$ with $a_{n}=a_{n}^{*}\left(e^{1 / \alpha}-1\right)^{-1}, b_{n}=G(\log n / n)-a_{n}$.

Theorem 4. In order that $F \in \mathscr{D}_{m M}\left(L_{2 a}^{*}\right)$ with $a_{n}=a_{n}^{*}\left(1-e^{-1 / \alpha}\right)^{-1}$, it is necessary that

$$
\lim r_{n}(\beta, \delta)=e^{-\delta / \alpha}\left(e^{-\beta / \alpha}-1\right)\left(e^{-1 / \alpha}-1\right)^{-1}
$$

for all real $\delta, \beta$ and $\epsilon_{n}=o(1)=\epsilon_{n}^{\prime}$. Conversely, if $\lim r_{n}(\beta, \delta)$ exists $\left({ }^{4}\right)$ for all real $\delta, \beta$ and $\epsilon_{n}^{\prime}=o(1)=\epsilon_{n}$ and if for some $\alpha>0$ and all real $\delta$ and $\epsilon_{n}=o(1)=\epsilon_{n}^{\prime}$,

$$
\lim _{n \rightarrow \infty} r_{n}(1, \delta)=e^{-\delta / \alpha},
$$

then $F \in \mathscr{D}\left(L_{2 \alpha}^{*}\right)$ with $a_{n}=a_{n}^{*}\left(1-e^{-1 / \alpha}\right)^{-1}, b_{n}=G(\log n / n)+a_{n}$.

Proofs. The necessity of (2.17) or (2.18) follows directly from (2.6) and (2.11).

The proof of sufficiency for Theorem 4 requires only minor emmendations from that of Theorem 3 and therefore only the latter will be given. Set $a_{n}=a_{n}^{*}\left(e^{1 / \alpha}-1\right)^{-1}$. Then, as in the proof of Theorem 2, a simple consequence of $(2.17)^{\prime}$ is

(2.19) $\lim _{n \rightarrow \infty} \frac{1}{a_{n}}\left[G\left(\frac{\log n-\delta-k+\epsilon_{n}^{\prime}}{n}\right)-G\left(\frac{\log n-\delta+\epsilon_{n}}{n}\right)\right]=e^{k / \alpha}\left(e^{k / \alpha}-1\right)$ 
for all real $\delta$, all integers $k$ and all $\epsilon_{n}=o(1)=\epsilon_{n}^{\prime}$. Again, the case $k=0$ implies that $\lim r_{n}\left(\beta, \delta ; \epsilon_{n}, \epsilon_{n}^{\prime}\right)$ is independent of $\epsilon_{n}$ and $\epsilon_{n}^{\prime}$.

Exactly as in Theorem 2, lime $e^{-\delta / \alpha} r_{n}(\beta, \delta)$ is independent of $\delta$. Consequently, using also (2.19)

$$
\begin{aligned}
e^{\delta / \alpha}\left(e^{1 / \alpha}-1\right) & \\
= & \lim _{n \rightarrow \infty} \sum_{i=0}^{k-1} \frac{1}{a_{n}}\left[G\left(\frac{\log n-\delta-(i+1) / k}{n}\right)-G\left(\frac{\log n-\delta-i / k}{n}\right)\right] \\
= & \sum_{i=0}^{k-1} \exp \left[\frac{\delta+i / k}{\alpha}\right] \\
& \cdot \lim _{n \rightarrow \infty} \frac{e^{-\delta / \alpha}}{a_{n}}\left[G\left(\frac{\log n-\delta-1 / k}{n}\right)-G\left(\frac{\log n-\delta}{n}\right)\right],
\end{aligned}
$$

implying for all positive integers $k$, real $\delta$ and $\epsilon_{n}=o(1)=\epsilon_{n}^{\prime}$ that

$$
\lim _{n \rightarrow \infty} \frac{1}{a_{n}}\left[G\left(\frac{\log n-\delta-1 / k+\epsilon_{n}^{\prime}}{n}\right)-G\left(\frac{\log n-\delta+\epsilon_{n}}{n}\right)\right]=e^{\delta / \alpha}\left(e^{1 / k \alpha}-1\right),
$$

which, in turn, readily yields

$$
\lim _{n \rightarrow \infty} \frac{1}{a_{n}}\left[G\left(\frac{\log n-\delta-r+\epsilon_{n}^{\prime}}{n}\right)-G\left(\frac{\log n-\delta+\epsilon_{n}}{n}\right)\right]=e^{\delta / \alpha}\left(e^{r / \alpha}-1\right)
$$

for all rational $r$, real $\delta$ and $\epsilon_{n}=o(1)=\epsilon_{n}^{\prime}$. This is extended to real $r$ exactly as in Theorem 2. From this extended version of (2.20), for $x$ real, all $\epsilon>0$ and $n>N_{\epsilon}$,

$$
e^{x / \alpha}-1-\epsilon<\frac{1}{a_{n}}\left[G\left(\frac{\log n-x+\epsilon_{n}}{n}\right)-G\left(\frac{\log n}{n}\right)\right]<e^{x / \alpha}-1+\epsilon .
$$

Setting $b_{n}=G(\log n / n)-a_{n}$, for all $\epsilon>0$ and $n>N_{\iota}$,

$$
a_{n}\left(e^{x / \alpha}-\epsilon\right)+b_{n}<G\left(\frac{\log n-x+\epsilon_{n}}{n}\right)<a_{n}\left(e^{x / \alpha}+\epsilon\right)+b_{n},
$$

which is tantamount to (2.1) with $\gamma(x)=\alpha \log x, \alpha>0, x>0$, and completes the proof.

In contradistinction to the case of the minimum of independent identically distributed random variables, virtually all the standard distributions (including the Cauchy) $\in \mathscr{D}_{m M}\left(L_{3}^{*}\right)$ if indeed there is a nondegenerate limit distribution. Notwithstanding, $\mathscr{D}_{m M}\left(L_{1 \alpha}^{*}\right)$ and $\mathscr{D}_{m M}\left(L_{2 \alpha}^{*}\right)$ are not empty as is seen from the following 
Example. Let the inverse of $1-F$ be defined by

$$
G(v)=c_{1} e^{\rho u^{2} / 2}+c_{2}, \quad u=-\log v+\left(1-\frac{1}{\log v}\right) \cdot \log (-\log v)
$$

where $c_{1}+c_{2}>0, \rho \neq 0$ and $v$ is small( $\left(^{5}\right)$. If $v=(\log n-\gamma) / n$, then $u=\log n$ $+(\gamma+o(1)) / \log n$, whence

$$
\begin{aligned}
G\left[\frac{\log n-\gamma}{n}\right] & =c_{1} \exp \left[\rho(\log n)^{2} / 2+\rho \gamma+o(1)\right]+c_{2} \\
& =a_{n}(x+o(1))+b_{n},
\end{aligned}
$$

where $a_{n}=\left|c_{1}\right| \exp \left[\rho(\log n)^{2} / 2\right], \quad b_{n}=c_{2}, \quad x=(\operatorname{sgn} \rho) \cdot \exp (\rho \gamma)$ and $c_{1}<0$, $c_{2}>0$ for $\rho<0$ while $c_{1}>0$ if $\rho>0$. Hence for sufficiently large $n$,

$$
1-F\left(a_{n} x+b_{n}\right)=\frac{\log n-\gamma+o(1)}{n} .
$$

Thus the limiting distribution of the normalized minimax is of the type $L_{1 \alpha}^{*}$ or $L_{2 \alpha}^{*}$ depending on the sign of $\rho$.

Next, we note a link between $\mathscr{D}_{M}\left(L_{3}\right)$ and $\mathscr{D}_{m M}\left(L_{3}^{*}\right)$. If $F \in \mathscr{D}_{M}\left(L_{3}\right)$, then as in Lemma 1 , for all real $x[3]$,

$$
F\left(a_{n}^{\prime} x+b_{n}^{\prime}\right)=1-\frac{1}{n} e^{-x}+o\left(n^{-1}\right) .
$$

Suppose that (2.23) continues to hold for $x_{n}=x / \log n-\log \log n$, i.e.,

$$
\begin{aligned}
& F\left(a_{n}^{\prime}\left(\frac{x}{\log n}-\log \log n\right)+b_{n}^{\prime}\right) \\
& \quad=1-\frac{1}{n} \exp \left\{-\left(\frac{x}{\log n}-\log \log n\right)\right\}+o\left(n^{-1}\right) .
\end{aligned}
$$

Then, setting

$$
\begin{aligned}
a_{n} & =a_{n}^{\prime}(\log n)^{-1}, b_{n}=b_{n}^{\prime}-a_{n}^{\prime} \log \log n, \\
F\left(a_{n} x+b_{n}\right) & =1-\frac{\log n}{n}\left[1-\frac{x}{\log n}+o\left(\frac{1}{\log n}\right)\right]+o\left(n^{-1}\right), \\
& =1-\frac{\log n}{n}+\frac{x}{n}+o\left(n^{-1}\right),
\end{aligned}
$$

so that $F \in \mathscr{D}_{m M}\left(L_{3}^{*}\right)$ according to (2.1) and (2.11).

On the other hand, (2.24) may be contravened and (2.25) fail to be the correct normalization even though the domains of attraction are unchanged. For example if $F(x)=1-\exp \{-x /(1-x)\}, 0<x<1$, then, as noted

$\left(^{5}\right)$ The nature of $G(v)$ for $v$ bounded away from zero is unimportant from the standpoint of which limit distribution $F$ is attracted to. Thus (as may be necessary for monotonicity), $G$ may be modified for $v$ bounded sufficiently far from zero. 
in [3], $F \in \mathscr{D}_{M}\left(L_{3}\right)$ with $a_{n}^{\prime}=(\log n)^{2}, \dot{b}_{n}^{\prime}=(1+\log n)^{-1} \log n$. However, $F \in \mathscr{D}_{m M}\left(L_{3}^{*}\right)$ with

$$
\begin{aligned}
& a_{n}=(1+\log n-\log \log n)^{-2}(\log n)^{-1}, \\
& b_{n}=(1+\log n-\log \log n)^{-1}(\log n-\log \log n) .
\end{aligned}
$$

3. Asymptotic independence of minimax $X_{i j}$ and maximin $X_{i j}$. The joint distribution of $W_{n}$ and $Z_{n}$ is easy to obtain in principle but difficult to analyze. Let $u<v$ and $A_{s}^{(n)}$ denote the event that all rows of $\chi_{n}$ in (1.2) have an element greater than $v$ and all columns have an element less than or equal to $u$ while exactly $s$ of the $n^{2}$ elements are no greater than $u$ and exactly $t$ of the elements are greater than $v$. Then

$$
\begin{aligned}
P\left\{Z_{n} \leqq u, W_{n}>v\right\} & =\sum_{s=n}^{n^{2}-n} \sum_{t=n}^{n^{2}-s} P\left\{A_{s t}^{(n)}\right\} \\
& =\sum_{s=n}^{n^{2}-n} \sum_{t=n}^{n^{2}-s} a_{s t}^{(n)} F^{s}(u)[1-F(v)]^{t}[F(v)-F(u)]^{n^{2}-s-t}
\end{aligned}
$$

where $a_{s t}^{(n)}$ is the number of ways of arranging $s$ white balls, $t$ red balls and $n^{2}-s-t$ green balls in an $n \times n$ square array in which each column has at least one white ball and each row at least one red ball.

Equation (3.1) does not seem amenable to establishing the asymptotic independence of $W_{n}$ and $Z_{n}$. On the other hand it hints at the feasibility of an "order statistics" approach. For this reason we shall first concern ourselves with $\min _{i} \max _{j} Y_{i j}$ and $\max _{j} \min _{i} Y_{i j}$ where the $Y_{i j}$ are obtained by assigning the integers 1 to $n^{2}$ at random among the $n^{2}$ positions of the $n \times n$ matrix. The study of this minimax and maximin will be facilitated by the use of a construction which simultaneously yields the random variables $X_{i j}, Y_{i j}$ and related random variables, whose distributions are linked to those occurring in the classical occupancy problem [2].

The construction is rather long and will be divided into several stages. Two of these stages will then be reexamined in more detail.

STAGE 1 . Let the integer 1 be assigned to one of the $n^{2}$ positions $(i, j)$, $1 \leqq i \leqq n, 1 \leqq j \leqq n$, at random. Select one of the remaining $n^{2}-1$ positions at random to hold the integer 2 . Continue in this fashion until each column has at least one element and let $S$ be the number of integers thus required. The first $S$ integers will be called "little."

Stage 2. Assign the integer $n^{2}$ to one of the remaining $n^{2}-S$ positions at random. Then select one of the remaining $n^{2}-S-1$ positions to hold $n^{2}-1$. Continue until each row has at least one of the elements $n^{2}, n^{2}-1, \ldots$ or until all $n^{2}-S$ remaining integers are exhausted. Let $T$ be the number of integers thus required. These integers will be called "big." 
Stage 3. Assign the remaining $n^{2}-S-T$ positions (if any) at random to the remaining integers. Define $Y_{i j}$ to be the integer in position $(i, j)$ and note that

$$
\begin{aligned}
& \max _{j} \min _{i} Y_{i j}=S, \\
& \min _{i} \max _{j} Y_{i j}=n^{2}-T+1 \text { if } S+T<n^{2} .
\end{aligned}
$$

Define $T^{*}$ by

$$
\min _{i} \max _{j} Y_{i j}=n^{2}-T^{*}+1
$$

and observe that $T^{*}=T$ if $S+T<n^{2}$ and $T^{*} \geqq T$ in general.

StaGe 4. If $U$ is uniformly distributed on $(0,1), x=F^{-1}(U)$, where $F^{-1}$ is an inverse of $F$, has the c.d.f. $F$. If $F^{-1}$ is not unique select it to be compatible with $G$, i.e., $F^{-1}(y)=G(1-y)$. Now select $n^{2}$ independent observations on $U$ and let $X_{i}=F^{-1}\left(U_{i}\right)$ where the $U_{i}$ is the $i$ th ordered observations. Thus $U_{1} \leqq U_{2} \leqq \cdots \leqq U_{n^{2}}$ and $X_{1} \leqq X_{2} \leqq \cdots \leqq X_{n^{2}}$. Let $X_{i j}=X_{Y_{i j}}$. Then

$$
\begin{aligned}
& \max _{j} \min _{i} X_{i j}=X_{S}=F^{-1}\left(U_{S}\right), \\
& \min _{i} \max _{j} X_{i j}=X_{n^{2}-T^{*}+1}=G\left(1-U_{n^{2}-T^{*}+1}\right) .
\end{aligned}
$$

The random variables $X_{i j}$ so constructed are independent, indentically distributed with common c.d.f. $F$.

We now elaborate on Stage 1. First note that if exactly $i$ columns are occupied when $r$ integers have been placed, the probability that $r+1$ will be in a new column is $n(n-i) /\left(n^{2}-r\right) \geqq(n-i) / n$. Let $U_{i s}$ be independent and uniformly distributed on $(0,1), 1 \leqq i \leqq n, s \geqq 1$.

We shall select the positions of the "little" numbers via the random variables $U_{i s}$. Concomitantly, the $U_{i s}$ will be used for two related occupancy problems. We proceed as follows.

Divide $(0,1)$ into $n^{2}$ equal intervals corresponding to the $n^{2}$ positions. Let the interval into which $U_{11}$ falls determine the position $(i, j)$ of the integer 1 . Then, redivide $(0,1)$ into $n^{2}-1$ equal intervals, the first $n(n-1)$ of which correspond to positions in the unoccupied columns and the remainder to the other vacant positions. Let the value of $U_{21}$ determine the position of the integer 2. However, if it falls in an unoccupied column, i.e., $U_{21} \leqq p_{21}$ $=n(n-1) /\left(n^{2}-1\right)$, use $U_{31}$ to determine the position of the integer 3 ; if 2 lands in an already occupied column, i.e., $U_{21}>p_{21}$, use $U_{22}$ to locate the position of 3 . Continue inductively. If $U_{i s}$ has assigned the integer $r$ to an unoccupied column, divide $(0,1)$ into $n^{2}-r$ equal intervals, the first $n(n-i)$ of which correspond to the positions in the unoccupied columns while the others correspond to the remaining vacant positions. In this case, $U_{i+1,1}$ is 
used to determine the position of the integer $r+1$. On the other hand, if $U_{i s}$ has consigned $r$ to an already occupied column, again divide $(0,1)$ into $n^{2}-r$ equal intervals but now let the first $n(n-i+1)$ of these correspond to the positions in the vacant columns while the others correspond to remaining unoccupied positions. In this case, let $U_{i, s+1}$ determine the position of $r+1$.

Then $S=S_{1}+S_{2}+\cdots+S_{n}$ where $S_{1}+S_{2}+\cdots+S_{i}$ is the integer whose placement first leads to the occupation of $i$ columns. If we have observed $r$ integers occupying $i$ columns prior to the observations of $U_{i+1, s}$, it will lead to an $(i+1)$ st column if $U_{i+1, s} \leqq p_{i+1, s}=n(n-i) /\left(n^{2}-r\right)$. Note that the $p_{i+1, s}$ are random, $p_{i+1, s} \geqq(n-i) / n$ and that $S_{i}$ is the smallest subscript $s$ for which $U_{i s} \leqq p_{i s}$.

In the classical occupancy problems, successive observations fall into one of $n$ cells independently and with equal probability. When $i$ cells have been occupied, the probability that the next observation will occupy an $(i+1)$ st cell is $p_{i+1}^{(1)}=(n-i) / n$. In such an experiment let $S^{(1)}=S_{1}^{(1)}+S_{2}^{(1)}$ $+\cdots+S_{n}^{(1)}$ where $S_{1}^{(1)}+\cdots+S_{i}^{(1)}$ is the number of observations taken when $i$ cells first become occupied. The same uniformly distributed variables $U_{i s}, 1 \leqq i \leqq n, s \geqq 1$ are used to construct $S_{i}^{(1)}$ as follows. Let $S_{i}^{(1)}$ be the smallest subscript for which $U_{i s} \leqq p_{i}^{(1)}=(n-i+1) / n$. We also define $S_{i}^{(2)}$ as the smallest subscript $s$ for which $U_{i s} \leqq p_{i}^{(2)}=(n-i+1) /(n-3 \log n)$ and $S^{(2)}=S_{1}^{(2)}+S_{2}^{(2)}+\cdots+S_{n}^{(2)}$.

The second stage may be elaborated similarly using uniformly and independently distributed random variables $V_{i t}$ where $T=T_{1}+T_{2}+\cdots+T_{n}$, $T_{i}$ is the smallest subscript $t$ for which $V_{i t} \leqq q_{i t}=m_{i t} /\left(n^{2}-r_{i t}\right)$, where $m_{i t}$ is the number of unoccupied cells in the $(n-i+1)$ rows which are still unoccupied by "big" numbers and $r_{i t}$ is the total number of positions occupied when $V_{i t}$ is observed. Let $q_{i}^{(1)}=p_{i}^{(1)}, q_{i}^{(2)}=p_{i}^{(2)}, q_{i}^{(3)}=(n-i+1)$ $(n-9 \log n) / n^{2}$, and let $T_{i}^{())}$be the smallest subscript $t$ for which $V_{i t} \leqq q_{i}^{())}$ and $T^{())}=\sum_{i=1}^{n} T_{i}^{(1)}$. Note that $q_{i}^{(3)}<q_{i}^{(1)}<q_{i}^{(2)}$. If $S+T<3 n \log n, p_{i}^{(1)} \leqq p_{\text {is }}$ $\leqq p_{i}^{(2)}$ and $q_{i t} \leqq q_{i}^{(2)}$. If no row has more than $9 \log n$ "little" elements, $q_{i}^{(3)} \leqq q_{i t}$.

In outline, the derivation of the asympt')tic distribution of $(S, T)$ consists of showing that $S+T=2 n \log n+O_{p}(n)$ and that with probability approaching one no row contains more than $9 \log n$ "little" elements. Thus with large probability $S$ is sandwiched between $S^{(1)}$ and $S^{(2)}$ which are relatively close together. Similarly $T$ and $T^{(1)}$ are sandwiched between $T^{(2)}$ and $T^{(3)}$. But $S^{(1)}$ and $T^{(1)}$ are independent and their asymptotic distributions are related to the solution of the occupancy problem.

We commence the derivation by presenting some immediate consequences of the construction.

(i) If $p_{i}^{(1)} \leqq p_{i s} \leqq p_{i}^{(2)}, S_{i}^{(1)} \geqq S_{i} \geqq S_{i}^{(2)}$. If $q_{i}^{(3)} \leqq q_{i t} \leqq q_{i}^{(2)}, T_{i}^{(3)} \geqq T_{i} \geqq T_{i}^{(2)}$. Since $q_{i}^{(3)}<q_{i}^{(1)}<q_{i}^{(2)}, T_{i}^{(3)} \geqq T_{i}^{(1)} \geqq T_{i}^{(2)}$. 
(ii) The $T_{i}^{())}$and $S_{i}^{())}$have geometric distributions. Each $S_{i}^{())}$is independent of each $T_{i^{\prime}}^{\left(j^{\prime}\right)}$. Furthermore $E\left(S^{(1)}\right)=\sum_{i=1}^{n-1} n /(n-i)=n[\log n+O(1)]$ and $\sigma_{S^{(1)}}^{2} \sim n^{2}$.

(iii) $S_{i}^{(1)}=S_{i}^{(2)}+\Delta_{i}$, where $\Delta_{i} \geqq 0, P\left\{\Delta_{i}=0\right\}=p_{i}^{(1)} / p_{i}^{(2)}$, and

$$
P\left\{\Delta_{i}=r\right\}=\frac{p_{i}^{(2)}-p_{i}^{(1)}}{p_{i}^{(2)}}\left(1-p_{i}^{(1)}\right)^{r-1} p_{i}^{(1)}, \quad r \geqq 1 .
$$

(iv) $T_{i}^{(3)}=T_{i}^{(2)}+\Xi_{i}$, where $\Xi_{i} \geqq 0, P\left\{\Xi_{i}=0\right\}=q_{i}^{(3)} / q_{i}^{(2)}$, and

$$
P\left\{\Xi_{i}=r\right\}=\frac{q_{i}^{(2)}-q_{i}^{(3)}}{q_{i}^{(2)}}\left(1-q_{i}^{(3)}\right)^{r-1} q_{i}^{(3)}, \quad r \geqq 1 .
$$

\section{LEMMA 2.}

$$
\begin{aligned}
& \Delta=\sum_{i=1}^{n} \Delta_{i}=O_{p}(\log n)^{2}, \\
& \Xi=\sum_{i=1}^{n} \Xi_{i}=O_{p}(\log n)^{2} .
\end{aligned}
$$

\section{Proof.}

$$
\begin{aligned}
& E\left(\Delta_{i}\right)=\frac{p_{i}^{(2)}-p_{i}^{(1)}}{p_{i}^{(2)}} \sum_{r=1}^{\infty} r p_{i}^{(1)}\left[1-p_{i}^{(1)}\right]^{r-1}=\frac{p_{i}^{(2)}-p_{i}^{(1)}}{p_{i}^{(2)} p_{i}^{(1)}}, \\
& E(\Delta)=\sum_{i=1}^{n} \frac{3 \log n}{n-i+1}=O(\log n)^{2} .
\end{aligned}
$$

Since $\Delta \geqq 0$, it follows that $\Delta=O_{p}(\log n)^{2}$. Similarly we find

$$
\begin{gathered}
E\left(\Xi_{i}\right)=\frac{q_{i}^{(2)}-q_{i}^{(3)}}{q_{i}^{(2)} q_{i}^{(3)}}, \\
E(\Xi)=\frac{12 n \log n-27(\log n)^{2}}{n-9 \log n} \sum_{i=1}^{n} \frac{1}{n-i+1}=O(\log n)^{2}
\end{gathered}
$$

and

$$
\Xi=O_{p}(\log n)^{2}
$$

LEMma 3.

$$
\mathscr{Y}\left(\frac{S^{(1)}-n \log n}{n}, \frac{T^{(1)}-n \log n}{n}\right) \rightarrow L_{3} \times L_{3}
$$

Proof. According to known results on the classical occupancy problem [2], the probability of occupying all $n$ cells with $n[\log n+x]$ observations approaches $\exp \left(-e^{-x}\right)$. Hence 


$$
P\left\{S^{(1)} \leqq n[\log n+x]\right\} \rightarrow L_{3}(x) .
$$

Since $S^{(1)}$ and $T^{(1)}$ are independent and identically distributed, the lemma follows.

To prepare for the next step we digress with a minor technical lemma.

LEMMA 4. If $X$ has the binomial distribution $\mathscr{B}\left([a m \log m], m^{-1}\right), a \geqq 1$, $m \geqq 1$ then $P\{X \geqq 4 a \log m\}=o\left(m^{-2 a}\right)$ as $m \rightarrow \infty$.

Proof. Since [1], $P\{X \geqq b\} \leqq E\left[e^{t(X-b)}\right]$ for $t>0$,

$$
\begin{aligned}
P\{X \geqq 4 a \log m\} & \leqq E\{\exp [X-4 a \log m]\} \\
& =\left[\frac{e}{m}+\left(1-\frac{1}{m}\right)\right][a m \log m] e^{-4 a \log m} \\
& \leqq \exp \left\{a m \log m\left[\log \left(1+\frac{e-1}{m}\right)-\frac{4}{m}\right]\right\}=o\left(m^{-2 a}\right) .
\end{aligned}
$$

LEMma 5. With probability approaching one, no row has more than $9 \log n$ "little" elements.

Proof. Consider the first $M=2 n \log n$ positions selected at random from the $n \times n$ matrix. After all of these are selected, the probability that the next will be in the first row is no greater than $n /\left(n^{2}-2 n \log n\right)$. Hence, in the course of assigning the first $M$ positions, the number of occupied cells in the first row is less than a variable with the binomial distribution $\mathscr{B}\left(M, m^{-1}\right)$ where $m=n-2 \log n$. Applying Lemma 4 with $2<a<9 / 4$, the probability that the number of occupied positions in the first row exceeds $9 \log n$ is $o\left(n^{-4}\right)$. Let $A_{M},\left(A_{S}\right)$ be the event that some row contains more than $9 \log n$ of the first $M,(S)$ integers assigned. Then $P\left(A_{S}\right) \leqq P\left(A_{M}\right)$ $+P(S>M)$. But $P\left(A_{M}\right)=o\left(n^{-3}\right)$. From Lemma $3, S^{(1)}=n \log n+O_{p}(n)$. Since $p_{i}^{(1)} \leqq p_{i s}, S \leqq S^{(1)}$ and hence $P(S>M)=o(1)$. Thus $P\left(A_{S}\right) \rightarrow 0$ which is the desired result.

THEOREM 5. If the first $n^{2}$ integers are assigned at random to the positions of the $n \times n$ matrix yielding entries $Y_{i j}, S=\max _{j} \min _{i} Y_{i j}$, and $n^{2}-T^{*}+1$ $=\min _{i} \max _{j} Y_{i j}$, then $S=S^{(1)}+O_{p}(\log n)^{2}, T^{*}=T^{(1)}+O_{p}(\log n)^{2}$ where $S^{(1)}$ and $T^{(1)}$ are independent. Furthermore

$$
\lim _{n \rightarrow \infty} \mathscr{L}\left(\frac{S-n \log n}{n}, \frac{T^{*}-n \log n}{n}\right)=L_{3} \times L_{3} .
$$

Proof. With probability approaching one, $S^{(1)}<(3 / 2) n \log n$ in which case $p_{i}^{(1)} \leqq p_{i s} \leqq p_{i}^{(2)}$. Applying Lemma 5 , we have with probability approaching one, $q_{i}^{(3)} \leqq q_{i t}, T \leqq T^{(3)} \leqq(3 / 2) n \log n$ and $q_{i}^{(3)} \leqq q_{i t} \leqq q_{i}^{(2)}$. Thus with probability approaching one, $S^{(1)} \geqq S \geqq S^{(2)}, T^{(3)} \geqq T \geqq T^{(2)}$, and $T^{(3)} \geqq T^{(1)} \geqq T^{(2)}$. 
It follows from Lemma 2 that $S=S^{(1)}+O_{p}(\log n)^{2}, T=T^{(1)}+O_{p}(\log n)^{2}$, and $T^{*}=T$ in probability (i.e., with probability approaching one). Applying Lemma 3 completes the proof of Theorem 5 .

To study the asymptotic behavior of $\left(W_{n}, Z_{n}\right)$, we require a lemma which is essentially a corollary of $\$ 2$. Here we assume that $F \in \mathscr{D}_{m M}\left(L_{j}^{*}\right)$ for some $j, b_{n}, a_{n}>0$ and $F \in \mathscr{D}_{M m}\left(L_{k}\right)$ for some choice of $k, b_{n}^{\prime}, a_{n}^{\prime}>0$. Let $h(\gamma)$ and $\bar{h}(\gamma)$ be the corresponding functions.

LeMma 6. If $\gamma_{n}=O_{p}(1)$ as $n \rightarrow \infty$, and $F \in \mathscr{D}_{m M}\left(L_{j}^{*}\right) \cap \mathscr{D}_{M m}\left(L_{k}\right)$,

$$
\begin{aligned}
& \frac{1}{a_{n}}\left\{G\left[\frac{\log n-\gamma_{n}}{n}\right]-b_{n}\right\}=h\left(\gamma_{n}\right)+o_{p}(1), \\
& \frac{1}{a_{n}^{\prime}}\left\{F^{-1}\left[\frac{\log n+\gamma_{n}}{n}\right]-b_{n}^{\prime}\right\}=\bar{h}\left(\gamma_{n}\right)+o_{p}(1) .
\end{aligned}
$$

Proof. Equation (2.6) states the first result for fixed $\gamma_{n}=\gamma$. Since $h(\gamma)$ is continuous and monotone, any bounded $\gamma$ interval $I$ can be subdivided into a finite number of subintervals over each of which $h(\gamma)$ increases by less than є. There is an $N$ such that for $n>N$

$$
\left|\frac{1}{a_{n}}\left[G\left(\frac{\log n-\gamma}{n}\right)-b_{n}\right]-h(\gamma)\right|<\epsilon
$$

for the $\gamma$ which are end points of these intervals. Since $G$ and $h$ are monotone (3.3) holds with $\epsilon$ replaced by $2 \epsilon$ for all $\gamma$ on $I$ and $n>N$. It follows that for every deterministic sequence $\gamma_{n}=O(1)$, the first line of (3.2) holds with $o_{p}(1)$ replaced by $o(1)$. In view of the properties of the "in probability" calculus, $[1],[4],(3.2)$ is thus valid when $\gamma_{n}=O_{p}(1)$ is random. The second part of (3.2) follows in a similar fashion from the analogue of equation (2.6).

LEMMA 7.

$$
W_{n}=G\left(\frac{T^{*}+o_{p}(n)}{n^{2}}\right), \quad Z_{n}=F^{-1}\left(\frac{S+o_{p}(n)}{n^{2}}\right) .
$$

Proof. Given $S=s=n \log n+z n$, the $S$ th order statistic $U_{S}$ from the sample of $n^{2}$ observations on $U$ (see Stage 4) has mean $s / n^{2}$ and variance $s\left(n^{2}-s+1\right) /\left(n^{2}+1\right)^{2}\left(n^{2}+2\right)$. Applying the Chebyshev inequality and the fact that $S=n \log n+O_{p}(n)$ it follows that

$$
U_{S}-\frac{S}{n^{2}}=O_{p}\left[\frac{(n \log n)^{1 / 2}}{n^{2}}\right]=o_{p}\left(n^{-1}\right) .
$$

Hence, recalling the equations of Stage 4, 


$$
Z_{n}=F^{-1}\left(\frac{S+o_{p}(n)}{n^{2}}\right)
$$

The statement about $W_{n}$ follows similarly.

TheOREM 6. If

$$
\mathscr{L}\left(\frac{W_{n}-b_{n}}{a_{n}}\right) \rightarrow L^{*} \text { and } \mathscr{L}\left(\frac{Z_{n}-b_{n}^{\prime}}{a_{n}^{\prime}}\right) \rightarrow L,
$$

then

$$
\mathscr{L}\left(\frac{W_{n}-b_{n}}{a_{n}}, \frac{Z_{n}-b_{n}^{\prime}}{a_{n}^{\prime}}\right) \rightarrow L^{*} \times L
$$

Proof. First we note that the existence of the limits $L^{*}$ and $L$ implies the existence of corresponding $h(\gamma)$ and $\bar{h}(\gamma)$ and the relevance of Lemma 6 .

According to Lemma 7 and the fact that $T^{*}-T^{(1)}=O_{p}(\log n)^{2}$,

$$
W_{n}=G\left[\frac{T^{*}+o_{p}(n)}{n^{2}}\right]=G\left[\frac{T^{(1)}+o_{p}(n)}{n^{2}}\right] .
$$

Define $\gamma_{n}$ by setting $\left(\log n-\gamma_{n}\right) / n$ equal to $\left[T^{(1)}+o_{p}(n)\right] / n^{2}$ in the argument of $G$. Then $\gamma_{n}=O_{p}(1)$ and by Lemma 6

$$
\frac{W_{n}-b_{n}}{a_{n}}=h\left(\gamma_{n}\right)+o_{p}(1)=h\left[\frac{n \log n-T^{(1)}}{n}+o_{p}(1)\right]+o_{p}(1) .
$$

Since $h$ is uniformly continuous on bounded intervals and $\left(n \log n-T^{(1)}\right) / n$ $=O_{p}(1)$,

$$
h\left[\frac{n \log n-T^{(1)}}{n}+o_{p}(1)\right]=h\left[\frac{n \log n-T^{(1)}}{n}\right]+o_{p}(1) .
$$

Hence

$$
\frac{W_{n}-b_{n}}{a_{n}}=h\left(\frac{n \log n-T^{(1)}}{n}\right)+o_{p}(1)
$$

and

$$
\mathscr{L}\left\{h\left(\frac{n \log n-T^{(1)}}{n}\right)\right\} \rightarrow L^{*}
$$

Similarly

$$
\frac{Z_{n}-b_{n}^{\prime}}{a_{n}^{\prime}}=\bar{h}\left(\frac{S^{(1)}-n \log n}{n}\right)+o_{p}(1)
$$


and

$$
\mathscr{L}\left\{\bar{h}\left(\frac{S^{(1)}-n \log n}{n}\right)\right\} \rightarrow L .
$$

But $S^{(1)}$ and $T^{(1)}$ are independent. Hence

$$
\begin{gathered}
\mathscr{L}\left\{h\left(\frac{n \log n-T^{(1)}}{n}\right), \bar{h}\left(\frac{S^{(1)}-n \log n}{n}\right)\right\} \rightarrow L^{*} \times L, \\
\left(\frac{W_{n}-b_{n}}{a_{n}}, \frac{Z_{n}-b_{n}^{\prime}}{a_{n}^{\prime}}\right)=\left[h\left(\frac{n \log n-T^{(1)}}{n}\right), \bar{h}\left(\frac{S^{(1)}-n \log n}{n}\right)\right]+o_{p}(1)
\end{gathered}
$$

and Theorem 6 follows.

\section{REFERENCES}

1. H. Chernoff, Large sample theory: parametric case, Ann. Math. Statist. 27(1956), 1-22.

2. W. Feller, An introduction to probability theory and its applications, Wiley, New York, 1950.

3. B. V. Gnedenko, Sur la distribution limite du terme maximum d'une série aléatoire, Ann. of Math. (2) 44(1943), 423-453.

4. J. W. Pratt, On a general concept of "in probability", Ann. Math. Statist. 30(1959), 549-558

\section{Stanford University,}

Stanford, California

Purdue University,

LAFAYETTE, I NDIANA 